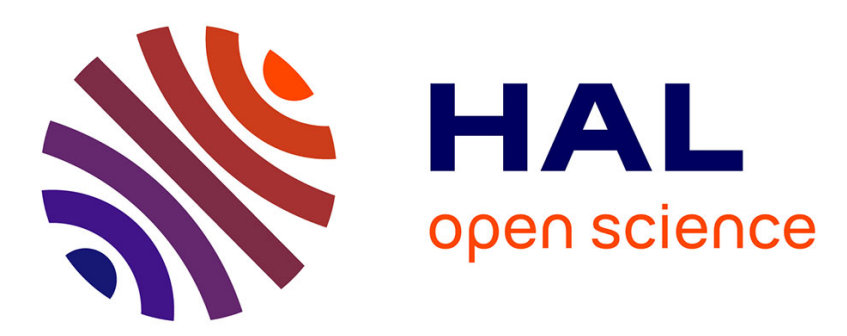

\title{
3D trajectory tracking control of quadrotor UAV with on-line disturbance compensation
}

\author{
Yasser Bouzid, Houria Siguerdidjane, Yasmina Bestaoui
}

\section{To cite this version:}

Yasser Bouzid, Houria Siguerdidjane, Yasmina Bestaoui. 3D trajectory tracking control of quadrotor UAV with on-line disturbance compensation. 1st IEEE Conference on Control Technology and Applications (CCTA 2017), Aug 2017, Mauna Lani, HI, United States. 10.1109/CCTA.2017.8062760 . hal-01722001

\section{HAL Id: hal-01722001 \\ https://hal.science/hal-01722001}

Submitted on 29 Sep 2020

HAL is a multi-disciplinary open access archive for the deposit and dissemination of scientific research documents, whether they are published or not. The documents may come from teaching and research institutions in France or abroad, or from public or private research centers.
L'archive ouverte pluridisciplinaire HAL, est destinée au dépôt et à la diffusion de documents scientifiques de niveau recherche, publiés ou non, émanant des établissements d'enseignement et de recherche français ou étrangers, des laboratoires publics ou privés. 


\title{
3D Trajectory Tracking Control of Quadrotor UAV with On-Line Disturbance Compensation
}

\author{
Y. Bouzid*, H. Siguerdidjane, Y. Bestaoui
}

\begin{abstract}
In this paper, we propose a revisited form of the so-called Model-Free Control (MFC). Herein, the MFC principle is employed to deal with the unknown part of the plant only (i.e. unmodeled dynamics, disturbances, etc.) and occurs beside an Interconnection and Damping Assignment-Passivity Based Control (IDA-PBC) strategy that is used instead of the PID structure as done in the classical MFC form. Using the proposed formulation, it is shown that we can significantly improve the performance of the control and its robustness level. This problem is studied in the case of Multi-Inputs Multi-Outputs (MIMO) system with an application to a small Vertical Take-Off and Landing (VTOL) vehicle where a stability analysis is also provided. The numerical simulations have shown satisfactory results where an in-depth discussion with respect to the control performance is highlighted by considering several scenarios and using several metrics.
\end{abstract}

\section{INTRODUCTION}

The quadrotors are considered as a good case study to design, to analyze and to implement flight control strategies. Moreover, it is necessary to design a controller such that the quadrotor will be able to efficiently follow a predefined trajectory, particularly in the presence of disturbances. For this reason, many studies have led to the development of sophisticated and robust nonlinear control laws (as for instance [1-3]). However, most of these proposed strategies require an accurate model in order to perform a good control, which is extremely difficult when the system is maneuvering in a harsh environment.

In this regards, a strategy based on a Model-Free technique is developed (MFC) (see as for instance [4]). The main advantage of this control strategy is that it does not require the knowledge of the system dynamics as it involves a continuous updating of the input-output of a very local model. Thus, its use as the basis of control allows the compensation of the uncertainties as well as other disturbances. It is employed in many real cases such as mobile robots [4] and quadrotors [5].

In a certain point of view, the control of a system with a model free has already been used, since many decades, on the basis of fuzzy logic control or the more popular one for linear systems through Ziegler-Nichols method [6]. In addition, assuming no available model is not totally a correct assumption due to the fact that most of systems, at least, may

\footnotetext{
*Y. Bouzid and Y. Bestaoui are with IBISC Laboratory, universitéd'Evry Val d'Essonne, Université Paris-Saclay, Evry, France (e-mail: yasseremp@gmil.com,Yasmina.Bestaoui@ufrst.univ-evry.fr).

H. Siguerdidjane iswith L2S, CentraleSupélec, Université Paris-Saclay, Gif sur yvette, France (e-mails:Houria.Siguerdidjane@,centralesupelec.fr).
}

be approximated by mathematical models even with poorly known dynamics.

Using the available information about the system will bring a notable benefit and significantly improve the performance of control. Therefore, we propose a Revisited Model-Free Control (R-MFC) strategy to simultaneously accommodate the unmodeled and neglected dynamics and external disturbances. As in general, the tuning of PID parameters allows to meet the desired specification of control, we use a reference modelbased control technique that achieves the control with the required specifications, by means of Interconnection and Damping Assignment-Passivity Based Control (IDA-PBC).

In the last two decades, the use of the so-called Port-Controlled Hamiltonian $(\mathrm{PCH})$ representation has attracted the attention of researchers. Many control tools have been developed to deal with this compact representation. Passivity-Based Control $(\mathrm{PBC})$ is well known especially in mechanical applications for controlling nonlinear systems. An improvement was developed through Interconnection and Damping Assignment (IDA) where the use of energy shaping was originated in [7]. Recently, the IDA-PBC has become an efficient tool in nonlinear control applications and has been illustrated in several real experimentations including electrical motors [8], magnetic suspension systems [9], etc.

Throughout this paper, a performance assessment is presented via results of several illustrations, scenarios and numerical simulations, with complementary comments of the proposed revisited strategy of control with respect to other techniques.

The remainder of this paper is organized as follows: Section II concerns the dynamics of the VTOL quadrotor and the control architecture. Section III and Section IV introduce the design of our nonlinear control approach. The simulation results are illustrated in Section V. Finally, the paper is ended with concluding remarks.

\section{QUADROTOR MODELING \& CONTROL ARCHITECTURE}

From the fundamental principle of dynamics, we model the quadrotor as a rigid body for the validation of control performance, neglecting some aerodynamic effects such as the gyroscopic and ground effects. The system operates in two coordinate frames: the Earth-fixed frame $R_{0}(O, X, Y, Z)$ and the body fixed frame $R_{1}\left(O_{1}, X_{1}, Y_{1}, Z_{1}\right)$ (see Figure 1). Let $\eta=$ $(\varphi, \theta, \Psi)^{T}$ describes the orientation of the aerial vehicle (Roll, Pitch, Yaw) and $\chi=(x, y, z)^{T}$ denotes its absolute position. 


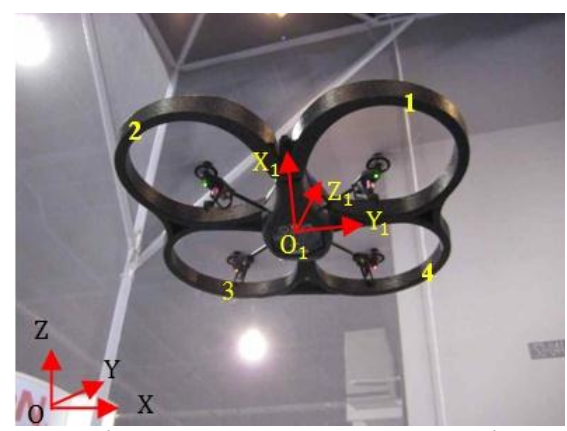

Figure 1. Frame representation.

In this study, we consider a simplified dynamic model of the vehicle that is derived in our previous work [1] in order to make the controller implementation simpler and easier and to show the efficiency of our control strategy that can deal with the unmodeled and neglected dynamics. The considered mathematical model may be written under the general mechanical equation, as follows

$\mathcal{M}(q) \ddot{q}+C(q, \dot{q}) \dot{q}+G=B(q) u$

where $q=[\chi, \eta]^{T} \in D_{\chi} \times D_{\eta} \subseteq \mathbb{R}^{3} \times \mathbb{R}^{3}$ is the generalized coordinates, $\mathcal{M}=\mathcal{M}^{T} \in \mathbb{R}^{6 \times 6}=\left[\begin{array}{cc}M & 0 \\ 0 & I\end{array}\right]>0$ is composed of the mass, $M=m I_{3 \times 3}$, and the inertia matrix, $C(q, \dot{q}) \in$ $\mathbb{R}^{6 \times 6}=\left[\begin{array}{cc}0_{3 \times 3} & 0_{3 \times 3} \\ 0_{3 \times 3} & \mathcal{C}\end{array}\right]$ denotes the Coriolis term, $G \in \mathbb{R}^{6}=[0,0, m g, 0,0,0]^{T}$ denotes the gravitational term, $B(q)=I_{6 \times 6}$ denotes the input matrix, with

$\mathcal{C} \in \mathbb{R}^{3 \times 3}=\frac{1}{2}\left[\begin{array}{ccc}0 & \left(I_{z}-I_{y}\right) \dot{\Psi} & \left(I_{z}-I_{y}\right) \dot{\theta} \\ \left(I_{x}-I_{z}\right) \dot{\Psi} & 0 & \left(I_{x}-I_{z}\right) \dot{\varphi} \\ \left(I_{y}-I_{x}\right) \dot{\theta} & \left(I_{y}-I_{x}\right) \dot{\varphi} & 0\end{array}\right]$

and

$u \in \mathbb{R}^{6}=\left[\begin{array}{c}u_{x} u_{1} \\ u_{y} u_{1} \\ u_{z} \\ u_{\varphi} \\ u_{\theta} \\ u_{\Psi}\end{array}\right]=\left[\begin{array}{c}\left(c_{\Psi} s_{\theta} c_{\varphi}+s_{\Psi} s_{\varphi}\right) u_{1} \\ \left(s_{\Psi} s_{\theta} c_{\varphi}-c_{\Psi} s_{\varphi}\right) u_{1} \\ \left(c_{\theta} c_{\varphi}\right) u_{1} \\ u_{2} \\ u_{3} \\ u_{4}\end{array}\right]$

with $m$ the mass, $g$ the gravity acceleration, $u_{1}$ the total thrust of four rotors, $\tau=\left(u_{2}, u_{3}, u_{4}\right)^{T}$ the control torque vector and $\mathrm{s}_{(.)}$and $\mathrm{c}_{(.)}$abbreviations for $\sin ($.$) and \cos ($.$) respectively.$ The parameters of the system UAV are displayed in Table 1.

Table 1. Quadrotor parameters.

\begin{tabular}{c|c|c|c}
\hline$m(k g)$ & 0.429 & $I_{y}\left(k g \cdot m^{2}\right)$ & 0.0029 \\
\hline$I_{x}\left(k g \cdot m^{2}\right)$ & 0.0022 & $I_{z}\left(k g . m^{2}\right)$ & 0.0048 \\
\hline
\end{tabular}

In order to simplify the design of the controller, two virtual inputs $u_{x}$ and $u_{y}$ are given as

$\left\{u_{x}=c_{\Psi} s_{\theta} c_{\varphi}+s_{\Psi} s_{\varphi}\right.$

$\left\{u_{y}=s_{\Psi} s_{\theta} c_{\varphi}-c_{\Psi} s_{\varphi}\right.$

The reference angles, $\varphi_{r} \neq \pm \frac{\pi}{2}$ and $\theta_{r} \neq \pm \frac{\pi}{2}$ are considered as inputs for the rotation subsystem. From system (4), it follows

$\left\{\begin{array}{c}\varphi_{r}=\arcsin \left(u_{x} \sin \Psi_{r}-u_{y} \cos \Psi_{r}\right) \\ \theta_{r}=\arcsin \left(\frac{u_{x} \cos \Psi_{r}+u_{y} \sin \Psi_{r}}{\cos \varphi_{r}}\right)\end{array}\right.$

\section{R-MFC FLIGHT CONTROLLER DESIGN}

The classic MFC approach proceeds by considering an ultralocal model, valid in short time that approximates the nonlinear model via input-output behavior using the experimental available data without any modeling step. However, this online numerical differentiator and estimation may fail with some highly nonlinear and/or time-varying dynamics that need to be treated carefully. In addition, most systems have a mathematical model even if it is not accurate. Note also that the MFC does not distinguish between model mismatches and perturbations. Therefore, to be more realistic, our approach employs the model-free principle to only deal with the unknown part of the controlled system where the use of the known part brings more benefit and makes the control more efficient.

This proposed formulation that we can denote by the acronym R-MFC is explained through a class of systems written under the general mechanical equation. This class of systems is widely adopted in the robotics and mechanical fields. For the sake of clarity, equation (1) is written under a compact form considering the general case

$\ddot{q}=\mathcal{F}(q, \dot{q})+\mathcal{B}(q) u$

where

$\mathcal{F}(q, \dot{q})=-\mathcal{M}(q)^{-1}(C(q, \dot{q}) \dot{q}+G)$ and $\mathcal{B}(q)=\mathcal{M}(q)^{-1} B(q)$ $q \in D_{q} \subseteq \mathbb{R}^{n}$ is the output vector, $\mathcal{M}=\mathcal{M}^{T} \in \mathbb{R}^{n \times n}>0$ is the inertia matrix, $C(q, \dot{q}) \in \mathbb{R}^{n \times n}$ denotes the Coriolis term, $G \in \mathbb{R}^{n}$ denotes the gravitational term, $B(q) \in \mathbb{R}^{n \times n}$ denotes the input matrix and $u \in \mathbb{R}^{n}$ denotes the input vector.

Usually, model (6) is quite simplified with neglected and unmodeled dynamics. Moreover, the execution of trajectories can be easily affected by external disturbances, which introduces some unknown terms. Therefore, a term $\delta \mathcal{F}$ could be added to nominal model (6) gathering the neglected and unmodeled dynamics and the external disturbances. An additional effort is requested to deal with this new term $\delta \mathcal{F}$. Thus, the input-output relationship of the anticipated model may be represented by the following system:

$q^{(v)}=\mathcal{F}(q, \dot{q})+\delta \mathcal{F}+\mathcal{B}(q) u+\kappa u$

where $\kappa \in \mathbb{R}^{n \times n}$ is a positive definite diagonal scale matrix fixed by the user and $\delta \mathcal{F}$ is an estimated term. In fact, the order $v=2$ of model (7) is chosen according to the prior knowledge of the system. It equals to the order of the mathematical model (6).

Remark 1: For the existing MFC technique, $v$ is fixed by the user and may equal to 1 or 2.

Therefore, the difference between model (6) and (7) lies in the presence of the unknown modeled part that can be estimated as

$\widehat{\delta \mathcal{F}}=\ddot{\hat{q}}-\mathcal{F}(q, \dot{q})-(\mathcal{B}(q)+\kappa) \hat{u}$

As the past input vector $\hat{u}$ in the previous time interval and the actual $2^{\text {nd }}$ derivative of the measured output vector $q$ are known, the value of $\widehat{\delta \mathcal{F}}$ is computed. This estimation is valid for a short period $T$ only and it should be continuously updated at every iteration of the closed loop controller. 
Obviously, the estimate of the second-order derivative $\ddot{\hat{q}}$ yields an estimate of $\widehat{\delta \mathcal{F}}$. Many significant advances on the numerical differentiation of noisy signals are elaborated in the literature [10]. This updated term $\widehat{\delta \mathcal{F}}$ captures the unknown dynamics of the system as well as the disturbances during each period $T$ and then brings the required changes in the control input by compensation. From (7), the control input for our R-MFC proposed strategy can be split into two parts: $u=(\mathcal{B}(q)+\kappa)^{-1}\left(-u_{C}+u_{a}\right)$ where the matrix $\mathcal{B}(q)+\kappa$ is non-singular in $D_{q}$.

$u_{C}=\widehat{\delta \mathcal{F}}$ plays the role of compensator of the unknown part and $u_{a}$ is considered as an auxiliary input that ensures the asymptotic convergence of the tracking errors of the closed loop into the origin. Injecting input (9) in (7), leads to a new and fully known nonlinear model eliminating the unknown part

$\ddot{q}=\mathcal{F}(q, \dot{q})+u_{a}$

The existing MFC technique employs, in the case of $v=2$, a PID controller as an auxiliary input :

$u_{a}=\ddot{q}_{r}+K_{I} \int_{t_{0}}^{t_{1}} E(\tau) d \tau+K_{P} E+K_{D} \dot{E}$

that leads to the intelligent PID where $E=q-q_{r}$ is the tracking error vector and $q_{r}$ is the reference trajectory vector. $K_{P}, K_{I}$ and $K_{D}$ denote the usual tuning gains.

Herein, we proceed with a different way by employing a sophisticated tool rather than the PID structure where the main goal is to ensure the asymptotic convergence, towards the origin, of the tracking errors of closed-loop of model (10). In this stage, a broad range of strategy can be applied.

The use of PID, in the classic form of MFC, allows to ensure a given performance of the system time response according to the given specifications (overshoot, settling time, accuracy) via the tuning of the control parameters. To achieve the desired specifications, by using another control procedure, is more challenging. Therefore, we employ a reference model based control strategy where the control specifications are a priori fixed. Then the control input pushes the system to follow the same behavior as the target model.

\section{IDA-PBC BASED AUXILIARY INPUT}

In the following, trajectory tracking control is achieved using the IDA-PBC approach. In this technique, we modify the total energy function of (10) to assign the desired equilibrium and damping injection matrix to meet the asymptotic stability. To preserve the energy interpretation, the closed-loop system is presented in a Port-Controlled Hamiltonian (PCH) representation.

\section{A. System energy and PCH model}

Explicitly, system (10) is written as

$\mathcal{M}(q) \ddot{q}+C(q, \dot{q}) \dot{q}+G=\mathcal{M}(q) u_{a}$

The system's Hamiltonian energy, $H(q, p)$, the sum of the kinetic energy, $T(q, p)$ and the potential one, $V(q)$, is written as:

$H(q, p)=T(q, p)+V(q)$
So,

$H(q, p)=\frac{1}{2} p^{T} \mathcal{M}^{-1}(q) p+G^{T} q$

where $p \in \mathbb{R}^{n}$ is the generalized momentum.

This system has a natural stable equilibrium configuration. This latter one is related to the minimum of energy. The $\mathrm{PCH}$ model is needed in order to design a controller based on the IDA-PBC methodology. From (12), the dynamic of the quadrotor can be written as

$\left[\begin{array}{c}\dot{q} \\ \dot{p}\end{array}\right]=[\mathcal{J}(q, p)-\mathcal{R}(q, p)]\left[\begin{array}{l}\nabla_{q} H(q, p) \\ \nabla_{p} H(q, p)\end{array}\right]+\left[\begin{array}{c}0 \\ \mathcal{M}(q)\end{array}\right] u_{a}$

with

$[\mathcal{J}(q, p)-\mathcal{R}(q, p)]=\left[\begin{array}{cc}0 & I_{6 \times 6} \\ -I_{6 \times 6} & -C(q, p)\end{array}\right]$

\section{B. Target dynamics}

Motivated by equation (13), we propose a desired energy function as being:

$H_{d}(q, p)=T_{d}(q, p)+V_{d}(q)$

We modify the total internal energy function of the closed loop system to assign the desired equilibrium configuration and we require that $H_{d}(q)$ will have a minimum at $\left(q^{*}, p^{*}\right)$, thus

$H_{d}(q, p)=\frac{1}{2}\left(p-p^{*}\right)^{T} \mathcal{M}_{d}^{-1}(q)\left(p-p^{*}\right)+V_{d}(q)$

where $V_{d}(q)$ and $\mathcal{M}_{d}=\mathcal{M}_{d}^{T}>0$ represent the desired closed loop potential energy function and inertia matrix, respectively with

$q^{*}=\arg _{\min } V_{d}(q)$

We take the reference trajectories as desired equilibrium configuration. In another words, $\left(q^{*}, p^{*}\right)=\left(q_{r}, p_{r}\right)$.

To preserve the energy interpretation we also require that the desired closed loop system be in $\mathrm{PCH}$ form

$\left[\begin{array}{c}\dot{q} \\ \dot{p}\end{array}\right]=\left[J_{d}(q, p)-\mathcal{R}_{d}(q, p)\right]\left[\begin{array}{l}\nabla_{q} H_{d}(q, p) \\ \nabla_{p} H_{d}(q, p)\end{array}\right]$

where

$J_{d}(q, p)=-J_{d}^{T}(q, p)=\left[\begin{array}{cc}0 & \mathcal{M}^{-1} \mathcal{M}_{d} \\ -\mathcal{M}^{-1} \mathcal{M}_{d} & J(q, p)\end{array}\right]$

$\mathcal{R}_{d}(q, p)=\mathcal{R}_{d}^{T}(q, p)=\left[\begin{array}{cc}0 & 0 \\ 0 & \mathcal{M}(q) K_{D I} \mathcal{M}(q)^{T}\end{array}\right]$

$J(q, p)$ is a skew symmetric matrix and $K_{D I}=K_{D I}^{T}>0$ contains the control parameters. Obviously, the matrix $\left[J_{d}(q, p)-\mathcal{R}_{d}(q, p)\right]$ has the same form as the original system.

\section{Energy shaping \& damping injection}

Commonly, the control input of IDA-PBC is decomposed into two terms [11].

$u_{a}=u_{E S}+u_{D I}$

where $u_{D I}$ acts on the damping and $u_{E S}$ is designed for the energy shaping.

The controller is obtained by substituting (23) in (15) and making the resulting equations equal to (20). Thus

$\left[\begin{array}{cc}0 & I_{6 \times 6} \\ -I_{6 \times 6} & -C\end{array}\right]\left[\begin{array}{l}\nabla_{q} H(q, p) \\ \nabla_{p} H(q, p)\end{array}\right]+\left[\begin{array}{c}0 \\ \mathcal{M}(q)\end{array}\right]\left(u_{E S}+u_{D I}\right)$ 


$$
=\left[\begin{array}{cc}
0 & \mathcal{M}^{-1} \mathcal{M}_{d} \\
-\mathcal{M}^{-1} \mathcal{M}_{d} & J(q, p)-\mathcal{M}(q) K \mathcal{M}(q)^{T}
\end{array}\right]\left[\begin{array}{l}
\nabla_{q} H_{d}(q, p) \\
\nabla_{p} H_{d}(q, p)
\end{array}\right]
$$

$\mathcal{R}_{d}$ injects a damping into the system via negative feedback of the passive output $\mathcal{M}^{T} \nabla_{p} H_{d}$. The damping injection term is then:

$u_{D I}=-K_{D I} \mathcal{M}(q)^{T} \nabla_{p} H_{d}(q, p)$

Thus, the second row leads to the energy shaping

$u_{E S}=\mathcal{M}(q)^{-1}\left(\nabla_{q} H(q, p)+C \nabla_{p} H(q, p)-\nabla_{q} H_{d}(q, p)+\right.$

$\left.J(q, p) \nabla_{p} H_{d}(q, p)\right)$

Finally, the R-MFC is split into three parts as

$u=(\mathcal{B}+\kappa)^{-1}\left(-u_{C}+u_{E S}+u_{D I}\right)$

where $u_{C}$ deals with the unknown parts and allows to maintain a certain level of robustness, $u_{E S}$ allows to meet the desired specification through the target model components and finally the damping injection term $u_{D I}$ in order to guarantee a damped response. Our approach is summarized by Figure 2 .

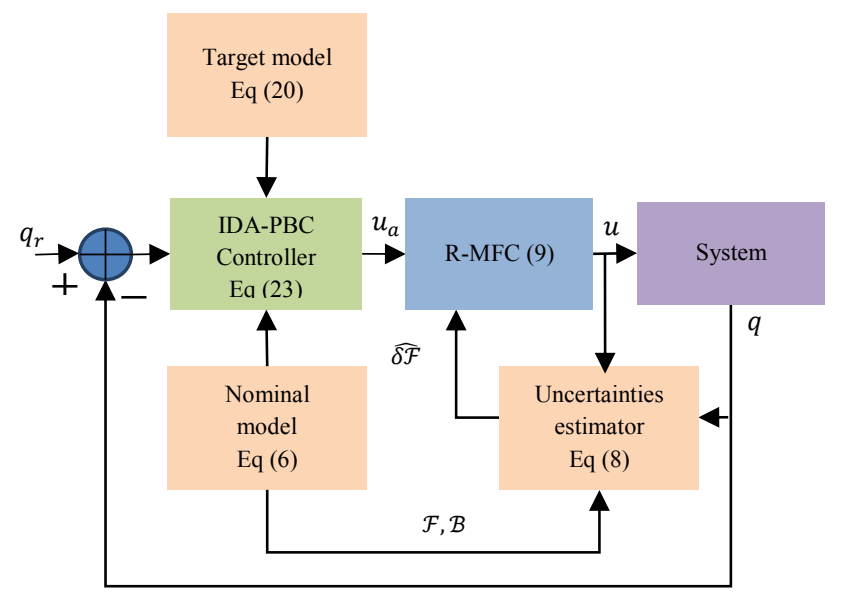

Figure 2. R-MFC scheme.

\section{Control design for quadrotor and stability analysis}

For the sake of simplification, we keep the interconnection matrix unchanged, namely $\mathcal{M}_{d}=\mathcal{M}$ and $J=0$, therefore $u_{E S}$ becomes

$u_{E S}=\mathcal{M}(q)^{-1}\left(\nabla_{q} H(q, p)+C \nabla_{p} H(q, p)-\nabla_{q} H_{d}(q, p)\right)$

So,

$u_{E S}=G+C \mathcal{M}^{-1} p-\nabla_{q} V_{d}(q)$

$V_{d}$ is an arbitrary function of $\chi$ and $\eta$. From equation (19), the necessary condition, $\nabla_{q} V_{d}\left(q^{*}\right)=0$ and the sufficient condition, $\partial_{q}^{2} V_{d}\left(q^{*}\right)>0$, will hold at $q^{*}$. $V_{d}$ has its minimum at $q^{*}=q_{r}$.

In our case we choose $V_{d}(q)$ to be a quadratic function, which leads to

$$
\begin{aligned}
V_{d}(q) & =\frac{1}{2}\left(\chi_{r}-\chi\right)^{T} K_{E S \chi}\left(\chi_{r}-\chi\right) \\
& +\frac{1}{2}\left(\eta_{r}-\eta\right)^{T} K_{E S \eta}\left(\eta_{r}-\eta\right)
\end{aligned}
$$

where $K_{E S_{\chi}}$ and $K_{E S_{\eta}}$ denote positive definite matrices of the control parameters.
Now, the auxiliary input for the quadrotor is given by

$u_{a}=u_{E S}+u_{I D}$

where

$u_{E S}=\left(\begin{array}{c}K_{E S_{\chi}}\left(\chi_{r}-\chi\right)+G \\ K_{E S_{\eta}}\left(\eta_{r}-\eta\right)+\mathcal{C} \dot{\eta}\end{array}\right)$ and $u_{D I}=K_{D I}\left(p_{r}-p\right)$

Proposition 1: if $\mathcal{M}_{d}=\mathcal{M}$ and $J=0$ with $V_{d}(q)$ having a quadratic form (30), the auxiliary input $u_{a}$ for the quadrotor, using IDA-PBC approach, can be given by (31).

The stability of the closed-loop dynamics of the quadrotor system in form (12) is introduced by the following theorem.

Theorem 1: Closed-loop of system (12) written under PCH form, using control law (31) is asymptotically stable.

Proof: Following the IDA-PBC approach described above, closed loop system (12) written under PCH model (15) is equivalent to the desired PCH model (20) using control law (31).

$H_{d}(q, p)$ is a positive definite function chosen herein as Lyapunov candidate function where the first time derivative is

$$
\begin{aligned}
\dot{H}_{d}(q, p)= & \dot{q}^{T} \nabla_{q} H_{d}(q, p)+\dot{p}^{T} \nabla_{p} H_{d}(q, p) \\
\text { Using }(29), \text { we get } & \dot{H}_{d}(q, p)=\nabla_{p} H_{d}(q, p)^{T} \nabla_{q} H_{d}(q, p)-\left(\nabla_{q} H_{d}(q, p)^{T}+\right. \\
& \left.\nabla_{p} H_{d}(q, p)^{T} \mathcal{M}^{T} K_{I D}^{T} \mathcal{M}\right) \nabla_{p} H_{d}(q, p)
\end{aligned}
$$

Then

$$
\begin{aligned}
\dot{H}_{d}(q, p) & =-\nabla_{p} H_{d}(q, p)^{T} \mathcal{M}^{T} K_{I D} \mathcal{M} \nabla_{p} H_{d}(q, p) \\
& \leq-e i g_{\min }\left(K_{I D}\right)\left(p-p_{r}\right)^{T}\left(p-p_{r}\right)
\end{aligned}
$$

So, closed loop of system (12) is asymptotically stable.

\section{RESUlTS AND DiscUSSION}

In this section, we test the effectiveness of the proposed controller not only in the ideal case but also in the presence of different disturbances. For the sake of further comparison, we follow the same protocol and fit the same conditions. The control parameters are tuned, using Genetic Algorithms (GA), in the ideal case then kept for the entire proposed scenarios and for which the objective is to reduce the steady state errors. Thus, the fitness function is given by

$$
I S E=\frac{1}{t_{f}-t_{i}} \int_{t_{i}}^{t_{f}}\left(\begin{array}{c}
\chi_{r}-\chi \\
\eta_{r}-\eta
\end{array}\right)^{T}\left(\begin{array}{c}
\chi_{r}-\chi \\
\eta_{r}-\eta
\end{array}\right) d t
$$

where $t_{i}$ and $t_{f}$ denote the initial and the final instants of optimization respectively. The obtained control parameters are depicted in Table 2.

Table 2: R-MFC control parameters.

\begin{tabular}{c|c|c|c|c|c}
\hline$K_{E S_{x}}$ & 23.33 & $K_{E S_{y}}$ & 22.19 & $K_{E S_{z}}$ & 21.77 \\
\hline$K_{D I_{x}}$ & 5.05 & $K_{D I_{y}}$ & 4.86 & $K_{D I_{z}}$ & 4.99 \\
\hline$K_{E S_{\varphi}}$ & 18.77 & $K_{E S_{\theta}}$ & 20.672 & $K_{E S_{\Psi}}$ & 19.16 \\
\hline$K_{D I_{\varphi}}$ & 4.51 & $K_{D I_{\theta}}$ & 5.00 & $K_{D I_{\Psi}}$ & 4.58 \\
\hline
\end{tabular}

For a significant analysis of the features of the proposed controller, two additional nonlinear controllers are considered. The first one is the classic model-free technique that exhibits 
promising results (for details one may refer to [5]) whilst the second one is traditionally applied for quadrotors i.e. Backstepping approach (BS).

The overall system dynamics and control laws are implemented using Matlab tool. The total simulation time is 40 seconds and the sampling time is set to 0.01 seconds. We simulate the response of the quadrotor using the available parameters of an AR-Drone (see Table 1). The following scenarios are successively proposed:

- Basic scenario: After the take-off, the quadrotor tracks a square reference trajectory $(2 m \times 2 m)$ described as

$\sigma(t)= \begin{cases}0 & \text { when } 0 \leq t \leq t_{1} \\ L_{r} \frac{\left(t-t_{1}\right)^{5}}{\left(t-t_{1}\right)^{5}+\left(T-t+t_{1}\right)} & \text { when } t_{1}<t \leq t_{2} \\ L_{r} & \text { when } t_{2}<t \leq t_{3} \\ L_{r}-L_{r} \frac{\left(t-t_{3}\right)^{5}}{\left(t-t_{3}\right)^{5}+\left(T-t+t_{3}\right)} & \text { when } t_{3}<t \leq t_{4} \\ 0 & \text { when } t_{4}<t \leq t_{f}\end{cases}$

with $T=5$ seconds, $t_{f}=40$ seconds and $L_{r}=2$ meters.

$x_{r}=\sigma(t)$ with $t_{1}=5, t_{2}=t_{1}+T, t_{3}=25, t_{4}=t_{3}+T$

$y_{r}=\sigma(t)$ with $\mathrm{t}_{1}=10, t_{2}=t_{1}+T, t_{3}=30, t_{4}=t_{3}+T$

$z_{r}=\sigma(t)$ with $t_{1}=0, t_{2}=t_{1}+T, t_{3}=35, t_{4}=t_{3}+T$

- Parameters uncertainties: We suppose that the inertia matrix elements and the aerodynamic coefficients are underrated by $50 \%$ of the real values.

- Extra Payload: The quadrotor is supposed carrying a heavy camera with an additional mass that represents $50 \%$ from the initial mass of quadrotor. The camera is modeled as a rigid compact body located at the center of mass of quadrotor.

- Sensor noise: We add the sensor noise on the states of the system. The expression of the noisy states is

$\int \tilde{\chi}=\chi+N_{c}$ rand $($.

$\left\{\tilde{\eta}=\eta+N_{c}\right.$ rand $()$.

rand(.) is a Matlab function, which generates a random number between 0 and $1 . N_{c}$ is a scale parameter to adjust the level of noise.

- Wind disturbance: We accept that the wind causes the same acceleration intensity on all X, Y,Z-axes. These accelerations are considered as perturbations added to the equations related to the forces in the quadrotor model. Therefore, the disturbed model may be expressed as follows

$\ddot{\tilde{x}}=\ddot{x}+a_{x}(t)$

$\ddot{\tilde{y}}=\ddot{y}+a_{y}(t)$

$\ddot{\tilde{z}}=\ddot{z}+a_{z}(t)$

The profile of this acceleration expressed explicitly as

$a_{i}(t)=0$

when $0 \leq t \leq t_{1}$

$a_{i}(t)=0.8 \sin \left(\frac{\pi(t-30)}{31}\right)+0.4 \sin \left(\frac{\pi(t-30)}{7}\right)$

$+0.08 \sin \left(\frac{\pi(t-30)}{2}\right)+0.056 \sin \left(\frac{\pi(t-30)}{11}\right)$ when $t_{1}<t \leq t_{2}$

$a_{i}(t)=0$

when $t_{2}<t \leq t_{f}$

with $t_{1}=10$ and $t_{2}=30 i=x, y, z$.
First of all, we start with the basic scenario. We plot separately, the tracking errors of the translations along $\mathrm{X}, \mathrm{Y}, \mathrm{Z}$-axes in Figure 3 and the control inputs in Figure 4.
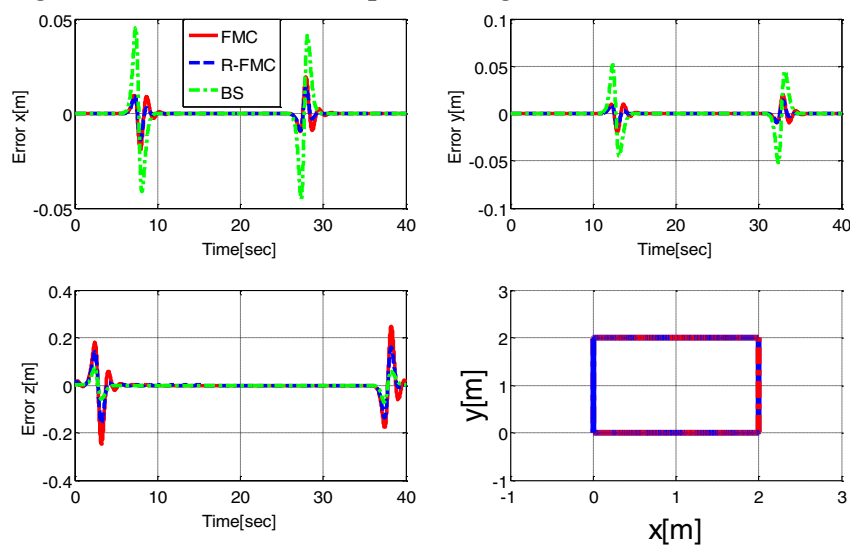

Figure 3. Tracking errors along the three axes.
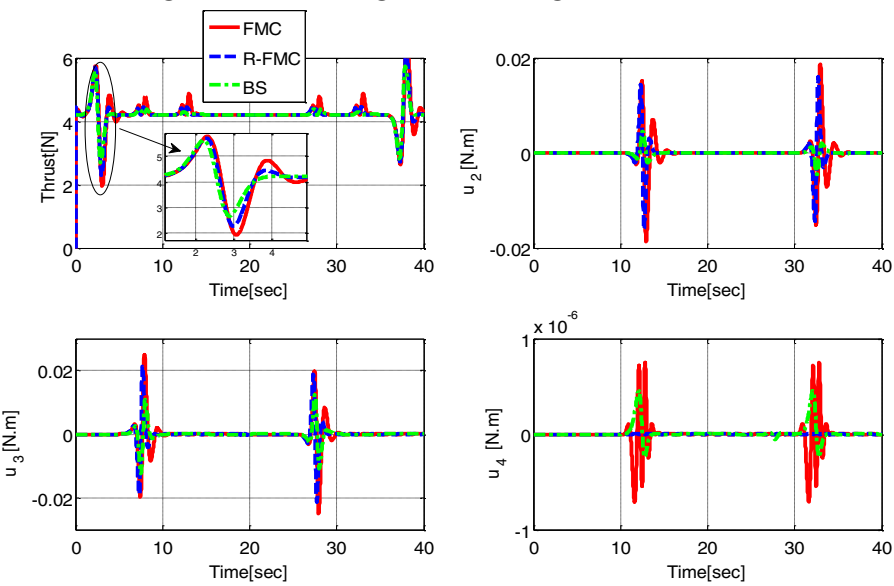

Figure 4. Control inputs.

Overall, from the curves obtained in Figures 3-4, the controllers stabilize correctly the roll, pitch and yaw angles where the quadrotor follows its reference trajectory with small errors with moderate energy consumption. From Figure 3, we observe that our proposed strategy ensures a damped response with less overshoot compared to the other considered techniques, which is an important result, due to the fact that the large overshoot leads to physical oscillations of the vehicle and engenders overturns nearby constraints.

We suggest quantifying the obtained results in order to get a close view of the features so deep analysis of the performance. Some analysis tools are considered such as:

- Integral Square Error (ISE): It is given by

$$
I S E=\int_{t_{0}}^{t_{f}}\left(e_{x}^{2}(t)+e_{y}^{2}(t)+e_{z}^{2}(t)\right) d t .
$$

- Integral Squared Control Input (ISCI): It allows to measure the consumed energy using $I S C I=\int_{t_{0}}^{t_{f}} u_{1}(t) d t$

The quantified metrics are summarized for each scenario in Table 3 .

Table 3: Metrics for each scenario.

\begin{tabular}{|c|c|c|c|}
\hline & BS & MFC [5] & R-MFC \\
\hline \multicolumn{4}{|c|}{ Basic scenario } \\
\hline ISE & 0.152 & 0.0884 & 0.0425 \\
\hline ISCI & 716.031 & 729.3093 & 719.76 \\
\hline
\end{tabular}




\begin{tabular}{|c|c|c|c|}
\hline \multicolumn{4}{|c|}{ Parameters uncertainties } \\
\hline ISE & 0.251 & 0.0955 & 0.0446 \\
\hline ISCI & 713.141 & 749.1154 & 721.9139 \\
\hline \multicolumn{4}{|c|}{ Extra payload } \\
\hline ISE & 0.767 & 0.0968 & 0.0451 \\
\hline ISCI & 1612.613 & $2.9240 \mathrm{e}+03$ & $2.8815 \mathrm{e}+03$ \\
\hline \multicolumn{4}{|c|}{ Sensors noise } \\
\hline ISE & 0.47 & 0.0950 & 0.0575 \\
\hline ISCI & 719.277 & 729.1249 & 719.8055 \\
\hline \multicolumn{4}{|c|}{ Wind gust } \\
\hline ISE & 0.28 & 0.0884 & 0.0425 \\
\hline ISCI & 675.040 & 688.0810 & 678.4928 \\
\hline
\end{tabular}

Overall, as illustrated in Table 3, the three controllers exhibit an acceptable behavior with moderate consumed energy regardless the external effect. Nonetheless, the extra payload as the gust of wind requests additional thrust in order to ensure good performance.

One may notice that, from Table 3 and for the basic scenario, the $\mathrm{BS}$ technique is the less accurate technique (ISE=0.152) followed by the classic MFC technique (ISE $=0.088$ ) while the $\mathrm{R}-\mathrm{MFC}$ is the most accurate techniques (ISE $=0.0425$ ). We also observe that the MFC consumes more energy while the R-MFC consumes less energy.

The accuracy of control degrades less and more for the BS according to the disturbance nature. However, the accuracy is almost the same using the MFC or R-MFC, which demonstrate the efficiency of the online estimation of the disturbance. It is then worthwhile to note that the MFC, as well as R-MFC, are insensitive to the disturbances where the thrust is requested according to the disturbance intensity in order to keep the same performance.

Finally, among the considered approaches, R-MFC exhibits the best performance in terms of accuracy and damped response with an acceptable level of energy consumption. Using our R-MFC is more relevant because that allows estimating the disturbances that affect our system as the wind, unlike the classic MFC that cannot distinguish the disturbances from the system dynamics.

\section{CONCLUSION}

In this paper, a relevant way to reformulate the actual MFC controller by R-MFC is described. It uses an auxiliary input and by bringing some changes (see Section III-IV), it operates in closed loop form. It improves the performance with respect to structured and unstructured uncertainties. Numerical simulations have been performed using the non-linear dynamic model of the quadrotor in order to test the effectiveness of the designed controller. The good efficiency of our approach is demonstrated in multiple test scenarios. The settling time is shown to be quite fast with good accuracy and a high level of robustness is ensured with respect to parameters uncertainties or external disturbances. The autonomous vehicle exhibits good performance under the wind gust and maintains its defined position very well. It is worthwhile to stress that our strategy is also able to estimate the disturbances. Some experimental tests will be reported in the near future in order to validate the obtained results.

\section{REFERENCES}

[1] Y. Bouzid, H. Siguerdidjane and Y. Bestaoui, "Improved 3D trajectory tracking by Nonlinear Internal ModelFeedback linearization control strategy for autonomous systems," 6th IFAC Symposium on System Structure and Control, Istanbul, 2016.

[2] Y. C. Choi and H. S. Ahn, "Nonlinear Control of Quadrotor for Point Tracking: Actual Implementation and Experimental Tests," IEEE/ASME Transactions on Mechatronics, vol. 20, no. 3, pp. 1179-1192, Jun. 2015.

[3] W. Zhu, H. Du, Y. Cheng, and Z. Chu, "Hovering control for quadrotor aircraft based on finite-time control algorithm," Nonlinear Dyn, pp. 1-11, Feb. 2017.

[4] L. x Ma, D. n Shi, M. x Chen, and X. q Wang, "Application of intelligent PID control for robot," in 2008 IEEE Conference on Cybernetics and Intelligent Systems, 2008, pp. 455-458.

[5] J. Wang, M. S. Geamanu, A. Cela, H. Mounier, and S. I. Niculescu, "Event driven model free control of quadrotor," in 2013 IEEE International Conference on Control Applications (CCA), 2013, pp. 722-727.

[6] K. J. Åström, C. C. Hang, and P. Persson, "Towards intelligent PID control," Annual Review in Automatic Programming, vol. 15, pp. 53-58, Jan. 1989.

[7] R. Ortega, A. v. d. Schaft, B. Maschke and G. Escobar, Stability and Stabilization of Nonlinear Systems, London; U.K: Springer-Verlag, 1999.

[8] H. González, M. A. Duarte-Mermoud, I. Pelissier, J. C. Travieso-Torres, and R. Ortega, "A novel induction motor control scheme using IDA-PBC," J. Control Theory Appl., vol. 6, no. 1, pp. 59-68, Feb. 2008.

[9] H. Rodriguez, H. Siguerdidjane and R. Ortega, "Experimental Comparison of Linear and Nonlinear Controllers for a Magnetic Suspension," Proceedings of the 2000 IEEE International Conference on Control Applications Anchorage, Alaska, USA, pp. 715-719, 2000.

[10] M. Mboup, C. Join, and M. Fliess, "Numerical differentiation with annihilators in noisy environment," Numer Algor, vol. 50, no. 4, pp. 439-467, Apr. 2009.

[11] R. Ortega, M. W. Spong, F. Gomez-Estern, and G. Blankenstein, "Stabilization of a class of underactuated mechanical systems via interconnection and damping assignment," IEEE Transactions on Automatic Control, vol. 47, no. 8, pp. 1218-1233, Aug. 2002. 